\title{
XMM-Newton detection of two clusters of galaxies with strong SPT Sunyaev-Zel'dovich effect signatures ${ }^{\star}$
}

\author{
R. Šuhada ${ }^{1}$, J. Song ${ }^{2}$, H. Böhringer ${ }^{1}$, B. A. Benson ${ }^{3,5,6}$, J. Mohr ${ }^{1,4,10}$, R. Fassbender ${ }^{1}$, A. Finoguenov ${ }^{1,9}$, D. Pierini ${ }^{1}$, \\ G. W. Pratt ${ }^{7}, \mathrm{~K}$. Andersson ${ }^{8}$, R. Armstrong ${ }^{2}$, and S. Desai ${ }^{2}$
}

1 Max-Planck-Institut für extraterrestrische Physik, Giessenbachstr. 1, 85748 Garching, Germany e-mail: rsuhada@mpe.mpg.de

2 University of Illinois, Department of Astronomy, 1002 West Green St, Urbana, IL 61801, USA

3 Kavli Institute for Cosmological Physics, University of Chicago, 5640 South Ellis Avenue, Chicago, IL 60637, USA

${ }^{4}$ Department of Physics, Ludwig-Maximilians-Universität, Scheinerstr. 1, 81679 Munich, Germany

5 Department of Physics, University of California, Berkeley, CA 94720, USA

${ }^{6}$ Enrico Fermi Institute, University of Chicago, 5640 South Ellis Avenue, Chicago, IL 60637, USA

7 Laboratoire AIM, IRFU/Service d'Astrophysique - CEA/DSM - CNRS - Université Paris Diderot, Bât. 709, CEA-Saclay, 91191 Gif-sur-Yvette Cedex, France

8 MKI, Massachusetts Institute of Technology, Cambridge, MA 02139, USA

9 University of Maryland, Baltimore County, 1000 Hilltop Circle, Baltimore, MD 21250, USA

10 Excellence Cluster Universe, Boltzmannstr. 2, 85748 Garching, Germany

Received 15 March 2010 / Accepted 14 April 2010

\section{ABSTRACT}

\begin{abstract}
We report on the discovery of two galaxy clusters, SPT-CL J2332-5358 and SPT-CL J2342-5411, in X-rays. These clusters were also independently detected through their Sunyaev-Zel'dovich effect by the South Pole Telescope, and in the optical band by the Southern Cosmology Survey. They are thus the first clusters detected under survey conditions by all major cluster search approaches. The X-ray detection is made within the frame of the XMM-BCS cluster survey utilizing a novel XMM-Newton mosaic-mode of observations. The present study makes the first scientific use of this operation mode. We estimate the X-ray spectroscopic temperature of SPT-CL J2332-5358 (at redshift $z=0.32$ ) to be $T=9.3_{-1.9}^{+3.3} \mathrm{keV}$, implying a high mass, $M_{500}=8.8 \pm 3.8 \times 10^{14} M_{\odot}$. For SPT-CL J2342-5411, at $z=1.08$, the available X-ray data do not allow us to directly estimate the temperature with good confidence. However, using our measured luminosity and scaling relations we estimate that $T=4.5 \pm 1.3 \mathrm{keV}$ and $M_{500}=1.9 \pm 0.8 \times 10^{14} M_{\odot}$. We find a good agreement between the X-ray masses and those estimated from the Sunyaev-Zel'dovich effect.
\end{abstract}

Key words. galaxies: clusters: individual: SPT-CL J2332-5358 - galaxies: clusters: individual: SPT-CL J2342-5411 - surveys $\mathrm{X}$-rays: galaxies: clusters

\section{Introduction}

Almost 40 years after the theoretical prediction of the SunyaevZel'dovich effect (SZE, Sunyaev \& Zeldovich 1972), i.e. the distortion of the cosmic microwave background spectrum by the hot gas in clusters of galaxies, we have entered a new era where the first clusters have been discovered by large-area SZE surveys (Staniszewski et al. 2009). Two ambitious SZE cluster surveys are currently underway: by the South Pole Telescope (SPT) and by the Atacama Cosmology Telescope (ACT). Recently, the SPT released a catalog of 21 SZE-selected galaxy clusters identified in the first $\sim 200 \mathrm{deg}^{2}$ of sky surveyed by the SPT (Vanderlinde et al. 2010, hereafter V10). Both SPT and ACT have additionally carried out observations of known clusters (Plagge et al. 2009; Hincks et al. 2009).

The SZE provides new prospects for precision cluster cosmology for two main reasons: (1) the SZE decrement characterized by the Comptonization parameter $\mathrm{Y}$ is currently considered as a robust, low-scatter proxy for cluster mass (e.g. da Silva et al. 2004; Motl et al. 2005) and (2) the SZE is not subject to the cosmological surface brightness dimming effect, resulting in

^ Appendix A is only available in electronic form at http: //www . aanda.org a selection function closely corresponding to a selection with a fixed mass limit at all redshifts.

However, to be able to fully harvest the potential of the upcoming comprehensive multi-wavelength surveys, we need to have (1) a good understanding of the cluster selection function, (2) cluster redshift measurements and (3) a well-calibrated link between cluster observables and total cluster masses.

In order to address these issues and to best understand the results of the different survey techniques, we are conducting a coordinated multi-wavelength survey in a test region (which will be covered by both SZE surveys) in the optical by the Blanco Cosmology Survey (BCS, $100 \mathrm{deg}^{2}$ ), in the mid-infrared with SPITZER $\left(14 \mathrm{deg}^{2}\right)$ and in X-rays with XMM-Newton.

Here we present the X-ray detections of two clusters, SPT-CL J2332-5358 and SPT-CL J2342-5411. These clusters were independently detected by SPT (V10) and confirmed to be coincident with overdensities of red galaxies (High et al. 2010). In addition, SPT-CL J2332-5358 has been recently detected in the optical (SCSO J233227-535827, Menanteau et al. 2010). This source is also coincident with the X-ray source 1RXS J233224.3-535840 in the ROSAT Bright Source Catalog (Voges et al. 1999). The present XMM-Newton observations enable us to confirm both objects as X-ray luminous clusters of galaxies. 

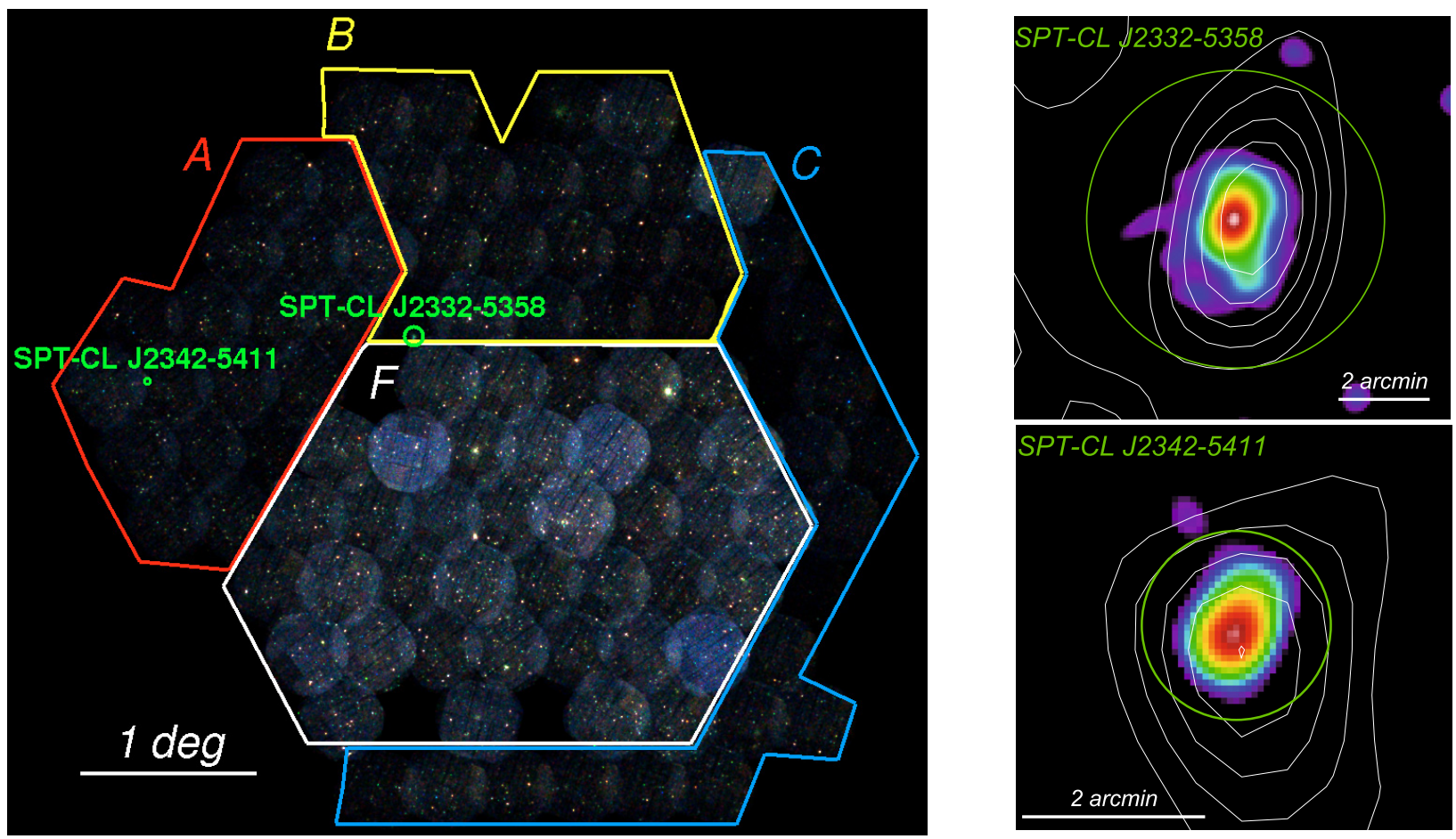

Fig. 1. Left: mosaic-mode XMM-Newton image of the whole $14 \mathrm{deg}^{2}$ survey field. The false color image was constructed from surface brightness images in the 0.3-0.5, 0.5-2.0 and 2.0-4.5 keV bands. Regions A, B, and C mark the three mosaic-mode observations, region F the deeper core of the survey consisting of 42 individual pointings. The green circles mark the positions of SPT-CL J2332-5358 and SPT-CL J2342-5411 with a radius equal to $\mathrm{r}_{\text {plat }}$ (Sect. 2.2) in both images. Right: 0.5-2.0 keV images of SPT-CL J2332-5358 (top) and SPT-CL J2342-5411 (bottom) with overlaid SZE signal-to-noise contours from the SPT survey (V10).

Throughout the article we adopt a $\Lambda$ CDM cosmology with $\left(\Omega_{\Lambda}, \Omega_{\mathrm{M}}, H_{0}\right)=\left(0.7,0.3,70 \mathrm{~km} \mathrm{~s}^{-1} \mathrm{Mpc}^{-1}\right)$.

\section{XMM-Newton data reduction}

The sources SPT-CL J2332-5358 and SPT-CL J2342-5411 were discovered as high-significance extended sources in $X M M-N e w t o n$ observations carried out in the framework of the XMM-BCS cluster survey (Šuhada et al., in prep.).

The X-ray survey currently extends over $14 \mathrm{deg}^{2}$ (Fig. 1). The core of the XMM-Newton field consists of a deeper region covering $6 \operatorname{deg}^{2}$ with 42 partially overlapping $\sim 12$ ks long individual pointings and three large scale $\left(\sim 2.7 \mathrm{deg}^{2}\right.$ each) mosaicmode observations. Each of the three mosaics consists of 19 stable pointings (3.5 ks exposures) and the slews between them, with a total time $\sim 90 \mathrm{ks}$ per mosaic.

\subsection{XMM-Newton mosaic-mode observations}

The mosaic-mode observation is a new observation mode of $X M M-N e w t o n$ and this is the first instance of its scientific use. Mosaic-mode observations were designed to significantly increase the efficiency of observations covering areas larger than the field of view of the telescope. Before the implementation of this mode such observations could only be achieved by consecutive independent single pointings. Each of these individual pointings then required its own instrumental overhead, which particularly for the EPIC pn camera can be a significant part of the total observing time, especially if the required exposure times for the pointings themselves are short.

The mosaic-mode observation starts as a standard observation with operational overhead (telescope pointing and guide star acquisition) followed by instrumental overhead, when a charge zero level (i.e. offset table) is calculated for the pn camera, which typically amounts to 3-4 ks (MOS cameras are operated with fixed offset tables and their setup is negligible). After the setups are finished, the observation itself starts. In our mosaics, each stable pointing has an exposure of $3.5 \mathrm{ks}$, followed by a slew to the next field offset by $\sim 23^{\prime}$. Science data are also collected during the slew and different from the standard operating mode the observation is not interrupted by a new instrumental setup sequence, but the same offset table is used during the whole mosaic.

Without the mosaic-mode, surveys of this kind would practically be unfeasible, with observing efficiency (i.e. the ratio of integration time to total time) around only $50 \%$, compared to $\gtrsim 80 \%$ efficiency achieved with the present setup. More information on the mosaic-mode observations can be found in the XMM-Newton User Handbook ${ }^{1}$.

\subsection{X-ray data analysis}

Both SPT-CL J2342-5411 and SPT-CL J2332-5358 were detected in the mosaic observations carried out in December 2009 (mosaic A, OBSID: 0604870301 and mosaic B, OBSID: 0604873401 respectively). We defer a more detailed description of the survey data reduction to a forthcoming publication of the $\mathrm{X}$-ray cluster catalog. Here we summarize the main steps and highlight the differences of treating mosaic eventlists with respect to standard observations.

The EPIC data were processed with the current XMM-Newton Standard Analysis System (SAS) version 9.0.0. We calibrated the raw observational data files in a standard way. Events in bad pixels, bad columns and close to the chip gaps were excluded from further analysis. The eventlists

\footnotetext{
1 xmm.esac.esa.int/external/xmm_user_support/ documentation/uhb/XMM_UHB.pdf
} 
were screened for high-background periods caused by soft proton flares following the two-step cleaning method of Pratt \& Arnaud (2003), but setting a $3 \sigma$ limit in both energy bands.

The clean exposure times are $71.9 / 72.6 \mathrm{ks}$ for $\mathrm{pn}$, and 85.0/88.7 ks for the MOS cameras for the entire mosaic A/B respectively. The beginning of the mosaic sequence $\mathrm{B}$ in the pn camera was strongly affected by soft proton flaring, therefore the effective exposure at the SPT-CL J2332-5358 location is only $0.1 \mathrm{ks}$ in pn, while it is $3.1 \mathrm{ks}$ in each MOS camera. The source in addition lies partially on the missing MOS1 CCD\#6, yielding a total combined MOS effective exposure of only $\sim 4 \mathrm{ks}$. Local exposure times for SPT-CL J2342-5411 were $\sim 2.8 \mathrm{ks}$ in pn, $2.1 \mathrm{ks}$ in MOS1 and $2.3 \mathrm{ks}$ in MOS2.

As the main source-detection algorithm we utilized the sliding box technique and a maximum likelihood source fitting in their current, improved implementation in the SAS tasks eboxdetect and emldetect.

Mosaic data of this extent is too extensive to fit into the memory storage during the detection process. Therefore we segmented the mosaic into several overlapping parts, which could be handled by the SAS tasks. Segmenting the mosaic into skychunks for source detection is preferable to splitting it into individual stable pointings, because we also wished to include counts gathered during the slews between the pointings and utilize the greater depth in the regions where two neighboring pointings overlap.

The mosaic segments have a typical size of $\sim 1 \mathrm{deg}^{2}$ and overlap by $\gtrsim 2^{\prime}$ along all borders. This way the input images, exposure, and background maps could be accommodated by the ebox - and emldetect tasks ran with increased memory buffer (imagebuffersize $=2000$ flag).

In order to get a reliable measurement of the flux and trace the emission of the clusters as far out as possible, we implemented a refined version of the growth curve method (Böhringer et al. 2000; Šuhada et al., in prep.). The cumulative source flux as a function of radius (i.e. the growth curves) for the two systems are displayed in Figs. A.1 and A.2 (in the online Appendix). The total source flux was determined iteratively by fitting a line to the flat part of the background-subtracted growth curve. We define the plateau radius $\left(r_{\text {plat }}\right)$ as the aperture where the growth curve reaches the total flux.

For SPT-CL J2332-5358 we detected source emission out to $r_{\text {plat }}=196^{\prime \prime}$, with total source flux of $F_{\text {plat }}(0.5-2.0 \mathrm{keV})=9.38 \pm$ $0.50 \times 10^{-13} \mathrm{erg} \mathrm{s}^{-1} \mathrm{~cm}^{-2}$, corresponding to a total luminosity $L_{\text {plat }}(0.5-2.0 \mathrm{keV})=2.67 \pm 0.14 \times 10^{44} \mathrm{erg} \mathrm{s}^{-1}$. Errors of the flux and luminosity include the Poisson errors and a $5 \%$ systematic error in the background estimation.

The X-ray morphology of this cluster agrees well with the SZE signal on the largest scales (Fig. 1) and its peak is close to the position of the brightest cluster galaxy (BCG, Fig. A.5). We detect a significant $X$-ray extension up to $\sim 1.5^{\prime} \mathrm{SE}$ from the BCG. A detailed characterization of the galaxy distribution and the correlation between X-ray and optical morphology will be given in a forthcoming paper.

For SPT-CL J2342-5411 we found $r_{\text {plat }}=62^{\prime \prime}, F_{\text {plat }}(0.5-$ $2.0 \mathrm{keV})=5.74 \pm 0.58 \times 10^{-14} \mathrm{erg} \mathrm{s}^{-1} \mathrm{~cm}^{-2}$ and a total luminosity $L_{\text {plat }}(0.5-2.0 \mathrm{keV})=2.84 \pm 0.3 \times 10^{44} \mathrm{erg} \mathrm{s}^{-1}$ (Fig. A.2).

\subsubsection{X-ray spectroscopy}

The available survey data $^{2}$, although modest in exposure, allow us to get a first temperature estimate for SPT-CL J2332-5358.

${ }^{2}$ Due to current limitations of the backscale task, used to calculate the area scaling factors of the spectra, we omitted the slew part
In order to determine a suitable aperture for spectroscopic measurements, we created a wavelet reconstruction (Vikhlinin et al. 1998) of the combined $0.5-2.0 \mathrm{keV}$ band image. We found that a circular aperture with $70^{\prime \prime}$ radius well encloses the region where the cluster emission is registered at $\geq 5 \sigma$ significance.

A background spectrum was extracted from an annulus concentric with the source and spanning the radial distance from $200^{\prime \prime}$ to $400^{\prime \prime}$. The inner radius was selected based on the growth-curve analysis as the radius where cluster emission is no longer observable (Fig. A.1). The outer radius is constrained by the field of view. We excised all detected point sources from each extracted spectrum after a visual check.

We fitted the spectrum with a single temperature MEKAL model, fixing the column density to the galactic value $n_{\mathrm{H}}=$ $1.62 \times 10^{20} \mathrm{~cm}^{-2}$ (Dickey \& Lockman 1990), metal abundance to $Z=0.3 Z_{\odot}$ and redshift to $z=0.32$ (photometric, High et al. 2010; consitent with Menanteau et al. 2010). To avoid biases stemming from analyzing low-count spectra, we used a minimally binned spectrum ( $\geq 1 \mathrm{cts} / \mathrm{bin})$ and C-statistics.

The fitted temperature is $T=9.3_{-1.9}^{+3.3} \mathrm{keV}$ ( $1 \sigma$ errors) for the joint fit from all three cameras (Fig. A.3). In order to check for possible systematics in the background subtraction, we also fitted the spectrum using background spectra extracted from a completely independent circular region (on different chips than the source but roughly at the same off-axis angle). The test background gives a consistent result, $T=9.4_{-1.9}^{+3.5} \mathrm{keV}$.

Based on our temperature measurement we estimated several important physical parameters (Table 1), including mass in $r_{500}$ and $r_{200}$ apertures from the $M-T$ scaling relation, assuming self-similar evolution. For parameters obtained from scaling relations we included the measurement errors of the luminosity and temperature and the intrinsic scatter of the scaling relations. We used a beta model to extrapolate the observed flux and luminosity out to $r_{500}$ (because the estimated $r_{500}$ value is higher than the measured $\left.r_{\text {plat }}\right)$. This extrapolation is negligible $(\sim 1.5 \%)$.

The available photon statistics for SPT-CL J2342-5411 is much lower and allows us to carry out a tentative analysis only. Following the previously described procedure, we extracted the source spectrum from a 45" region and the background spectrum from a concentric annulus with 100" inner and 200" outer radius. Fixing the column density to the galactic value $n_{\mathrm{H}}=$ $1.86 \times 10^{20} \mathrm{~cm}^{-2}$, metal abundance to $Z=0.3 Z_{\odot}$ and redshift to $z=1.08$ (photometric, High et al. 2010), we found that the spectrum is consistent with a single temperature MEKAL model (Fig. A.4). The temperature is only weakly constrained, $T=$ $6.7_{-2.4}^{+5.2} \mathrm{keV}$, and therefore we opted for the use of luminositybased scaling relations ( $L-T$ and $L-Y_{\mathrm{X}}$ ) to estimate the physical parameters of the system (Table 1 ). The evolution of the scaling relations and their intrinsic scatter is currently not firmly established out to $z \sim 1$. We assumed self-similar evolution of the scaling relations and no evolution of their intrinsic scatters. The error from these assumptions for SPT-CL J2342-5411 is expected to be smaller than the quoted measurement errors.

\section{Discussion and conclusions}

We have presented first results from the XMM-BCS cluster survey, providing X-ray detections of two SZE-selected systems, SPT-CL J2332-5358 and SPT-CL J2342-5411. The X-ray

of the survey for spectroscopical purposes and filtered from the mosaic eventlist only events detected during the relevant stable pointing period (the slew part would contribute only a few tens of counts in this case). 
A\&A 514, L3 (2010)

Table 1. Basic X-ray parameters of SPT-CL J2332-5358 and SPT-CL J2342-5411.

\begin{tabular}{llll}
\hline \hline Parameter & SPT-CL J2332-5358 & SPT-CL J2342-5411 & Units \\
\hline$\alpha(\mathrm{J} 2000)^{a}$ & $23^{\mathrm{h}} 32^{\mathrm{m}} 26.7^{\mathrm{s}}$ & $23^{\mathrm{h}} 42^{\mathrm{m}} 45.8^{\mathrm{s}}$ & \\
$\delta(\mathrm{J} 2000)^{a}$ & $-53^{\circ} 58^{\prime} 20.4^{\prime \prime}$ & $-54^{\circ} 10^{\prime} 59.2^{\prime \prime}$ & \\
photometric redshift & $0.32^{b}$ & $1.08^{b}$ & \\
$F_{500}[0.5-2.0 \mathrm{keV}]$ & $9.52 \pm 0.51$ & $0.58 \pm 0.06$ & $10^{-13} \mathrm{erg} \mathrm{cm}^{-2} \mathrm{~s}^{-1}$ \\
$L_{500}[0.5-2.0 \mathrm{keV}]$ & $2.71 \pm 0.15$ & $2.86 \pm 0.29$ & $10^{44} \mathrm{erg} \mathrm{s}^{-1}$ \\
$T_{500}$ & $9.3 \pm 2.6^{c}$ & $4.5 \pm 1.3^{f}$ & $\mathrm{keV}$ \\
$r_{500}$ & $1.3 \pm 0.2^{d}$ & $0.6 \pm 0.1^{d}$ & $\mathrm{Mpc}$ \\
$M_{500}$ & $8.8 \pm 3.8^{d}$ & $1.9 \pm 0.8^{d}$ & $10^{14} M_{\odot}$ \\
$Y_{\mathrm{X}, 500}$ & $11.6 \pm 9.7^{e}$ & $1.1 \pm 0.7^{g}$ & $10^{14} M_{\odot} \mathrm{keV}$ \\
$r_{200}$ & $2.0 \pm 0.3^{d}$ & $0.9 \pm 0.1^{d}$ & $\mathrm{Mpc}$ \\
$M_{200}$ & $12.4 \pm 5.4^{d}$ & $2.7 \pm 1.2^{d}$ & $10^{14} M_{\odot}$ \\
\hline
\end{tabular}

Notes. Flux and luminosity errors include the Poisson errors and a 5\% systematic error in the background estimation. Errors of parameters obtained from scaling relations include the measurement errors of the luminosity and temperature, respectively, and the intrinsic scatter of the scaling relations. We assume self-similar evolution for all the scaling relations and no evolution of their intrinsic scatters (see Sect. 2.2.1). (a) X-ray coordinates based on a maximum-likelihood fit of a PSF-folded beta model to the surface brightness distribution; ${ }^{(b)}$ High et al. (2010); ${ }^{(c)}$ spectroscopic temperature (Sect. 2.2.1), error bars averaged, assuming isothermality; ${ }^{(d)} M-T$ relation from Arnaud et al. (2005), using relations for $T>3.5 \mathrm{keV}$, self-similar evolution, radii calculated analytically from the mass estimates; ${ }^{(e)} M_{500}-Y_{\mathrm{X}}$ relation (Arnaud et al. 2007). ${ }^{(f)} L-T$ relation from Pratt et al. (2009), self-similar evolution, relation for the $0.5-2 \mathrm{keV}$ luminosity, BCES orthogonal fit; ${ }^{(g)} \quad L-Y_{\mathrm{X}}$ relation from Pratt et al. (2009), self-similar evolution, relation for the $0.5-2 \mathrm{keV}$ luminosity, BCES orthogonal fit. $Y_{X}$, the X-ray analogue to the Comptonization parameter $Y$, is the product of the gas mass and temperature (e.g. Kravtsov et al. 2006).

analysis is based on mosaic-mode XMM-Newton observations the first time observations of this kind have been carried out.

The system SPT-CL J2332-5358 ranks among the hottest known clusters $(T=9.3 \mathrm{keV})$ and is exceptionally massive $\left(M_{200} \gtrsim 1 \times 10^{15} M_{\odot}\right)$. Our mass estimate is in excellent agreement with the ROSAT-based result of Menanteau et al. (2010). The SZE-inferred mass reported by V10 is $M_{500}=5.20 \pm 0.86 \pm$ $0.83 \times 10^{14} M_{\odot}$, where the error bars represent the statistical and systematic uncertainties, both at $68 \%$ confidence. They note that this mass is biased low by a bright dusty point source identified in the $220 \mathrm{GHz}$ SPT data. A preliminary analysis indicates that this point source decreases the SZE mass estimate by a factor of $\sim 1.5$. This would imply a corrected SZE mass estimate of $M_{500}=7.8 \pm 1.3 \pm 1.3 \times 10^{14} M_{\odot}$, which agrees well with our $\mathrm{X}$-ray estimate.

This is an initial study of the system SPT-CL J2332-5358. Other aspects of the system are investigated in forthcoming papers: 1) using a deeper XMM observation Andersson et al. (in prep.) performing a detailed comparison of the X-ray and SZE properties of this cluster; 2) and a characterization of the galaxy population and morphology will be addressed in Song et al. (in prep.).

The source SPT-CL J2342-5411 belongs to one of the most distant known clusters $(z=1.08)$ with X-ray and SZE detections. The discovery of such a distant system in both SZE and X-ray surveys demonstrates the great potential of the two observational approaches for cosmological and cluster evolution studies. The estimated mass for this system, $M_{500}=1.9 \pm 0.8 \times 10^{14} M_{\odot}$, is consistent with the SZE mass $M_{500}=2.66 \pm 0.50 \pm 0.37 \times$ $10^{14} M_{\odot}(\mathrm{V} 10)$.

SPT-CL J2332-5358 and SPT-CL J2342-5411 are the first galaxy clusters discovered independently in X-ray, SZE and optical surveys. These clusters exemplify the promise of multiwavelength cluster surveys and give a glimpse of the possible synergies of current and future large-scale survey experiments, including SPT, Planck, eRosita, and the Dark Energy Survey.
Acknowledgements. We thank the XMM-Newton SOC for implementing the mosaic-mode. Particularly, we thank Pedro Rodriguez for useful discussions about the data analysis. We also thank the Blanco Cosmology Survey team for executing and processing the observations used in this paper. We thank the SPT team for SZE mass estimates and maps of this cluster. The South Pole Telescope is supported by the National Science Foundation through grants ANT-0638937 and ANT-0130612. The SPT also thanks the National Science Foundation (NSF) Office of Polar Programs, the United States Antarctic Program and the Raytheon Polar Services Company for their support of the project. R.S. acknowledges support by the DfG in the program SPP1177. H.B. acknowledges support for the research group through The Cluster of Excellence "Origin and Structure of the Universe", funded by the Excellence Initiative of the Federal Government of Germany, EXC project number 153. B.B. acknowledges additional support from a KICP Fellowship.

\section{References}

Arnaud, M., Pointecouteau, E., \& Pratt, G. W. 2005, A\&A, 441, 893 Arnaud, M., Pointecouteau, E., \& Pratt, G. W. 2007, A\&A, 474, L37 Böhringer, H., Voges, W., Huchra, J. P., et al. 2000, ApJS, 129, 435

da Silva, A. C., Kay, S. T., Liddle, A. R., \& Thomas, P. A. 2004, MNRAS, 348, 1401

Dickey, J. M., \& Lockman, F. J. 1990, ARA\&A, 28, 215

High, F. W., Stalder, B., Song, J., et al. 2010, ApJ, submitted [arXiv: 1003.0005 ]

Hincks, A. D., Acquaviva, V., Ade, P., et al. 2009, ApJ, submitted [arXiv: 0907.0461]

Kravtsov, A. V., Vikhlinin, A., \& Nagai, D. 2006, ApJ, 650, 128

Menanteau, F., Hughes, J. P., Barrientos, L. F., et al. 2010, ApJS, submitted [arXiv: 1002.2226]

Motl, P. M., Hallman, E. J., Burns, J. O., \& Norman, M. L. 2005, ApJ, 623, L63

Plagge, T., Benson, B. A., Ade, P. A. R., et al. 2009, ApJ, submitted [arXiv: 0911.2444]

Pratt, G. W., \& Arnaud, M. 2003, A\&A, 408, 1

Pratt, G. W., Croston, J. H., Arnaud, M., \& Böhringer, H. 2009, A\&A, 498, 361 Staniszewski, Z., Ade, P. A. R., Aird, K. A., et al. 2009, ApJ, 701, 32

Sunyaev, R. A., \& Zeldovich, Y. B. 1972, Comments Astrophys. Space Phys., 4, 173

Vanderlinde, K., Crawford, T. M., de Haan, T., et al. 2010, ApJ, submitted [arXiv: 1003.0003 ]

Vikhlinin, A., McNamara, B. R., Forman, W., et al. 1998, ApJ, 502, 558

Voges, W., Aschenbach, B., Boller, T., et al. 1999, A\&A, 349, 389

Pages 5 to 6 are available in the electronic edition of the journal at http: //www . aanda. org 


\section{Appendix A: Supplementary electronic material}

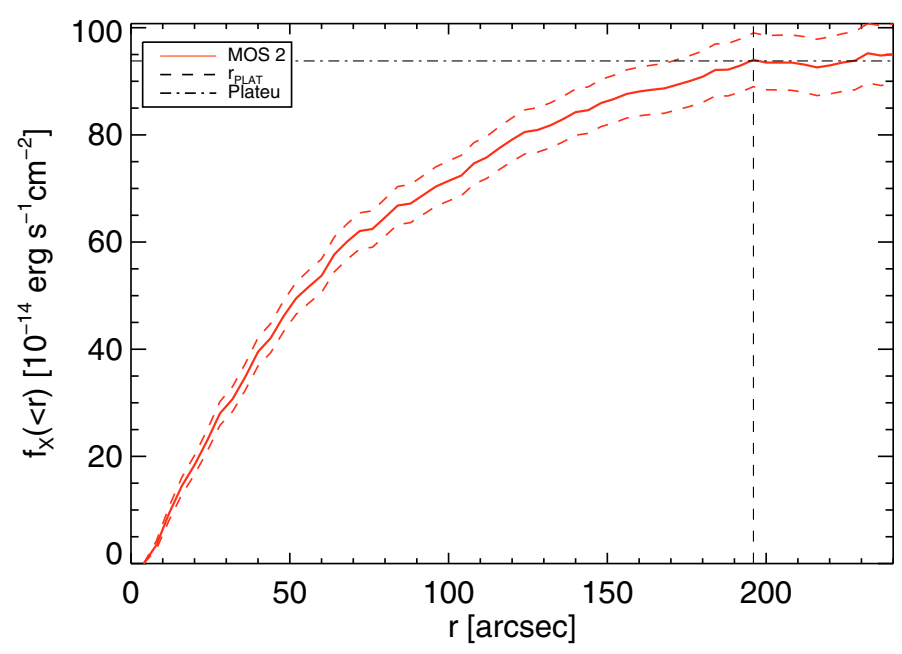

Fig. A.1. The growth curve of SPT-CL J2332-5358: red curve shows the integrated flux as a function of outer integration radius for the MOS2 camera in the $0.5-2.0 \mathrm{keV}$ band. Error bars on the growth curve indicate the Poisson error of the flux measurement including a 5\% systematic error in the background estimation. Source flux is detected out to $196^{\prime \prime}$ $\left(r_{\text {plat }}\right.$, dashed line). The total measured flux in this aperture and band is $F_{\text {plat }}=9.4 \pm 0.5 \times 10^{-13} \mathrm{erg} \mathrm{s}^{-1} \mathrm{~cm}^{-2}$ (horizontal dot-dashed level). We omitted the use of the pn and MOS1 camera for the growth curve analysis (see Sect. 2.2).

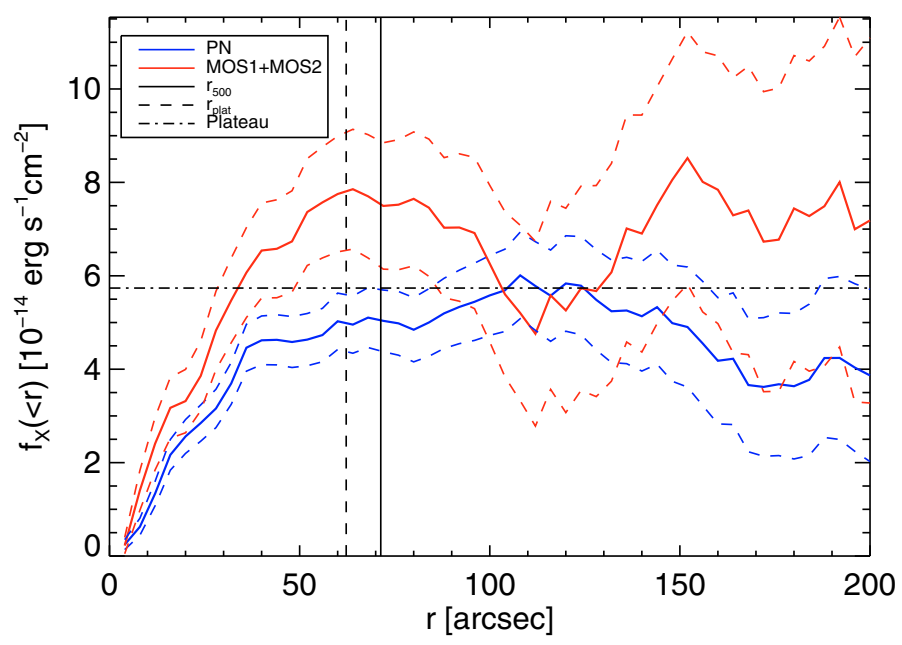

Fig. A.2. The growth curve of SPT-CL J2342-5411: red curve shows the integrated flux as a function of outer integration radius for the joint MOS1 and MOS2 cameras (0.5-2.0 keV band), blue curve for the pn camera. Error bars on the growth curve indicate the Poisson error of the flux measurement including a 5\% systematic error in the background estimation. Source flux is detected out to $r_{\text {plat }}=62^{\prime \prime}$ (dashed line). The total measured flux, estimated as the weighted average of the MOS and pn plateau fluxes, is $F_{\text {plat }}=5.7 \pm 0.6 \times 10^{-14} \mathrm{erg} \mathrm{s}^{-1} \mathrm{~cm}^{-2}$ (dot-dashed level).

The Appendix provides additional information concerning the X-ray analysis of SPT-CL J2332-5358 and SPT-CL J2342-5411.

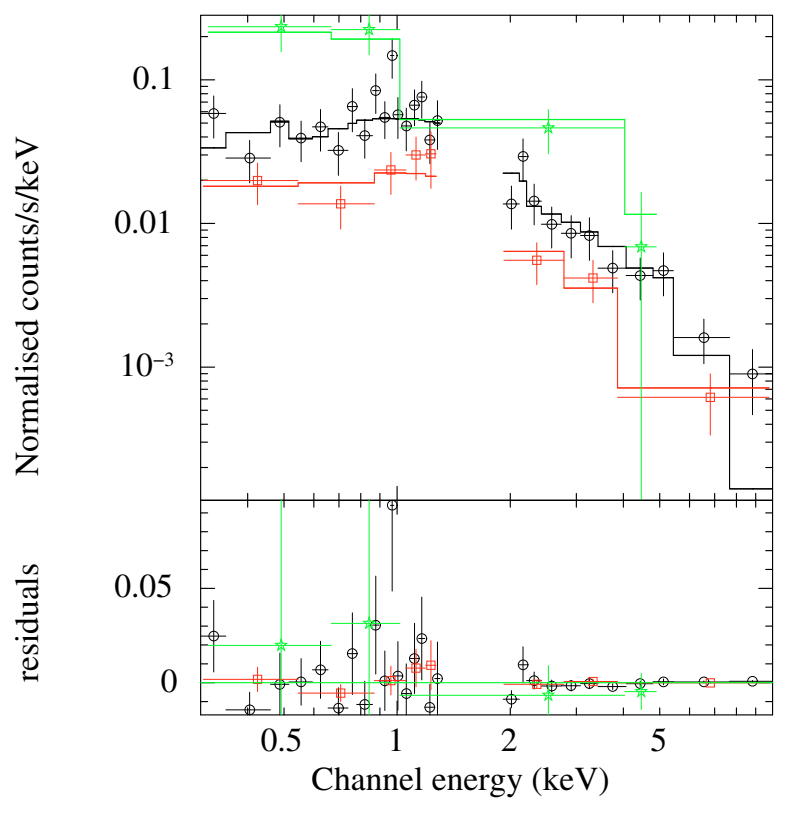

Fig. A.3. XMM-Newton X-ray spectrum of SPT-CL J2332-5358 fitted with a single temperature MEKAL model which gives $T=9.3_{-1.9}^{+3.3} \mathrm{keV}$. The column density is fixed to the galactic value $n_{\mathrm{H}}=1.62 \times 10^{20} \mathrm{~cm}^{-2}$ (Dickey \& Lockman 1990), the metal abundance to $Z=0.3 Z_{\odot}$ and the redshift to $z=0.32$ (photometric). The spectra were binned only for display purposes, the fit was carried out with $\geq 1 \mathrm{cts} /$ bin binning and C-statistic. Red: MOS1, black: MOS2, green: pn. See Sect. 2.2.1 for details.

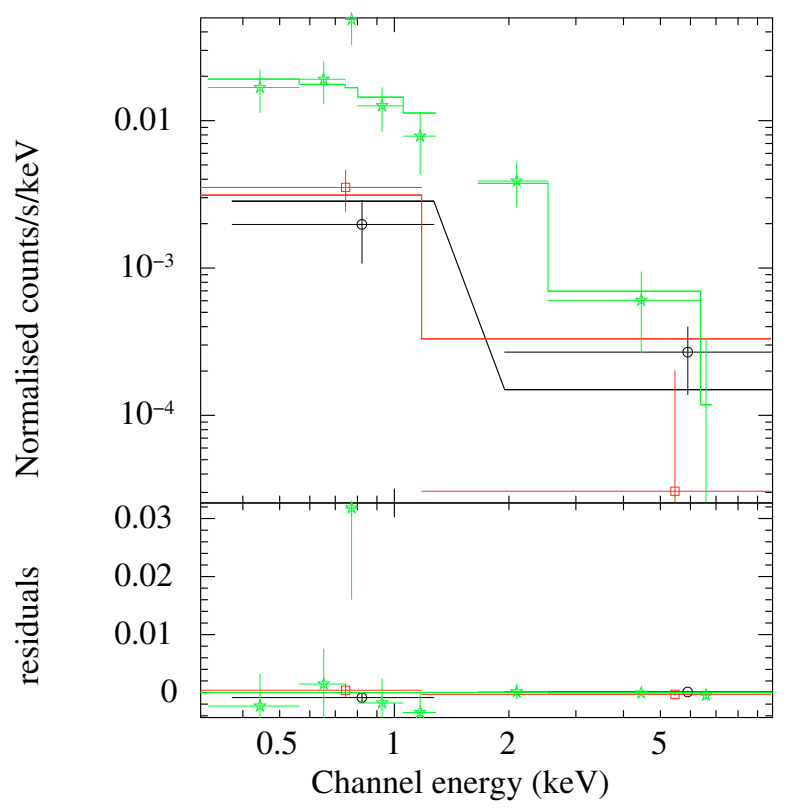

Fig. A.4. XMM-Newton X-ray spectrum of SPT-CL J2342-5411. The available low-count spectrum (displayed for completeness) allows us to draw only tentative conclusions. The spectrum is consistent with a single temperature MEKAL model, with the temperature only weakly constrained to $T=6.7_{-2.4}^{+5.2} \mathrm{keV}$. The column density is fixed to the galactic value $n_{\mathrm{H}}=1.86 \times 10^{20} \mathrm{~cm}^{-2}$ (Dickey \& Lockman 1990), the metal abundance to $Z=0.3 Z_{\odot}$ and the redshift to $z=1.08$ (photometric). The spectra were binned only for display purposes, the fit was carried out with $\geq 1 \mathrm{cts} /$ bin binning and C-statistic. Red: MOS1, black: MOS2, green: pn. See Sect. 2.2.1 for details. 

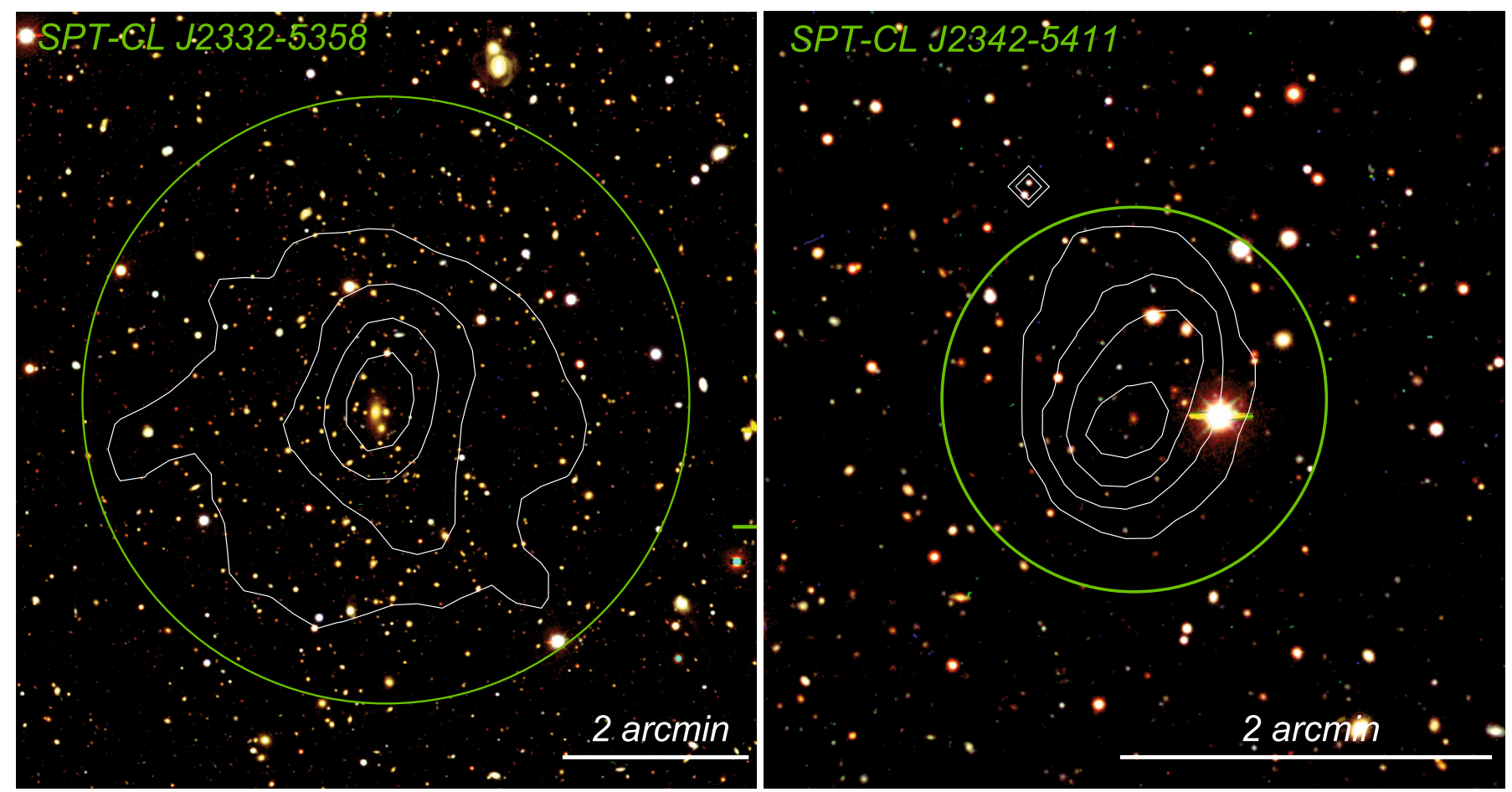

Fig. A.5. Left: color image of SPT-CL J2332-5358 (photo- $z=0.32$ ). Right: color image of SPT-CL J2342-5411 (photo- $z=1.08$ ). Both images were obtained from the Blanco Cosmology Survey imaging in the gri bands. X-ray contours are overlaid in white. Green circles show the estimated $r_{\text {plat }}$ (see Sect. 2.2). Both clusters have a large BCG (brightest cluster galaxy) within few arcseconds ( $\left.\$ 10^{\prime \prime}\right)$ from the X-ray emission peak. 